\title{
ИМПУЛЬСНЫЙ СТАБИЛИЗАТОР НАПРЯЖЕНИЯ С ЦИФРОВЫМ УПРАВЛЕНИЕМ ДЛЯ АВТОНОМНОЙ СИСТЕМЫ ЭЛЕКТРОПИТАНИЯ
}

\author{
Краснобаев Юрий Вадимович', \\ uvkras@mail.ru
}

\author{
Непомнящий Олег Владимирович', \\ 2955005@gmail.com
}

Иванчура Владимир Иванович', ivan43ura@yandex.ru

\section{Пожаркова Ирина Николаевна, ${ }^{2,}$ pozharkova@mail.ru}

\author{
Яблонский Алексей Павлович', \\ ehateslo@gmail.com \\ 1 Сибирский федеральный университет, \\ Россия, 660074, г. Красноярск, ул. Киренского, 26, корп. УЛК. \\ 2 Сибирская пожарно-спасательная академия ГПС МЧС России, \\ Россия, 662972, г. Железногорск, ул. Северная, 1
}

Актуальность. Результативность геофизических, геологоразведочных, метеорологических работ и работ по мониторингу окружающей среды во многом определяется системами электропитания испытательной и исследовательской аппаратуры. Специфика таких работ зачастую подразумевает использование полевых автономных систем. Первичными источниками энергии в таких системах, как правило, являются возобновляемые источники энергии, например солнечные батареи, ветро- или гидроэлектроустановки, а вторичными источниками энергии - аккумуляторные батареи. Источники энергии объединяются в систему электропитания посредством импульсных преобразователей энергии, которые выполняют функции по передаче энергии от её источников к потребителям, стабилизации напряжения на выходных шинах, предназначенных для питания потребителей и по повышению энергетической эффективности первичных источников энергии за счёт обеспечения их работы в режиме генерации максимальной мощности. Потребителем электроэнергии таких систем электропитания является сложная и разнородная аппаратура, часто имеющая импульсный характер энергопотребления, что приводит к значительным отклонениям напряжения на выходных шинах системы электропитания от стабильного уровня и, как следствие, к взаимному влиянию отдельных потребителей, приводящему к сбоям в их работе. Таким образом, импульсные преобразователи энергии должны обладать как способностью обеспечения работы первичных источников энергии в режиме генерации максимальной мощности, так и способностью обеспечивать режим стабилизации напряжения на выходных шинах. В режиме генерации максимальной мощности устройство управления импульсным преобразователем обеспечивает перевод и удержание рабочей точки на мощностной характеристике первичного источника в окрестности максимума мощности. В таком устройстве управления реализуется достаточно сложный алгоритм экстремального регулирования и на современном уровне развития техники устройство управления выполняется на основе программируемого цифрового устройства. Решение вопросов по применению этого же программируемого цифрового устройства и для цели управления импульсным преобразователем в режиме стабилизации напряжения на выходных шинах является актуальной задачей, поскольку позволит уменьшить количество элементов в устройстве управления преобразователем, что снизит его собственное энергопотребление и повысит надёжность функционирования.

Цель работы: решение теоретических и практических задач по обеспечению цифрового управления импульсным преобразователем в режиме стабилизации выходного напряжения с обеспечением малой длительности переходных процессов, вызванных приращениями тока нагрузки и астатизма выходного напряжения.

Методы: теория импульсных систем автоматического управления, математическое моделирование процессов в импульсных стабилизаторах напряжения и физическое макетирование.

Результаты. Проведен анализ особенностей автономных систем электропитания, синтезирован закон управления и разработана модель импульсного стабилизатора напряжения. Предложен метод и найдены способы управления импульсными стабилизаторами напряжения, которые обеспечивают малую длительность переходных процессов и астатизм выходного напряжения. Разработаны алгоритмы микропрограммного управления, реализующие найденные способы управления. Разработана архитектура и встроенное программное обеспечение для микропроцессорной системы управления стабилизатором. Изготовлен однокристальный вычислительный модуль устройства управления на базе программируемой интегральной схемы. Разработан и изготовлен макет импульсного стабилизатора с цифровым управлением на основе однокристального вычислительного модуля. Результаты экспериментального исследования макета подтверждают эффективность разработанного устройства управления, а именно достижение минимальной конечной длительности переходных процессов, вызванных ступенчатым изменением тока нагрузки, близкой к двум периодам преобразования и астатизма выходного напряжения. Показано, что применение импульсного стабилизатора, использующего полностью цифровой контур управления и реализуемого при помощи высокоскоростных микропроцессорных средств, имеет значительные преимущества по сравнению с аналоговыми вариантами.

\section{Ключевые слова:}

Возобновляемые источники энергии, автономная система электропитания, импульсный стабилизатор напряжения, цифровой контур управления, длительность переходного процесса, регулируемые составляющие переменных состояния. 


\section{Введение}

Для современной электронной аппаратуры, применяемой в составе геофизических, геологоразведочных, метеорологических комплексов и сложных систем мониторинга окружающей среды, характерным является эксплуатация в полевых условиях. Это подразумевает ее функционирование в автономном режиме с использованием энергии возобновляемых источников. Энергоэффективное производство и преобразование энергии на основе возобновляемых источников реализуется посредством систем электропитания (СЭП). В состав таких систем входят первичные источники энергии (ПИ), вторичные источники энергии аккумуляторные батареи (АБ), и энергопреобразующая аппаратура (ЭПА), состоящая из импульсных преобразователей энергии. В качестве ПИ энергии широко применяют возобновляемые источники энергии: солнечные батареи, ветро или гидроэлектроустановки $[1,2]$. Импульсные преобразователи ЭПА выполняют функции заряда АБ, и передачи энергии от первичных источников и АБ в нагрузку с одновременным обеспечением функции по экстремальному регулированию ПИ, что позволяет получить генерацию максимально возможной мощности первичными источниками [3]. Экстремальное регулирование осуществляется специальными импульсными преобразователями MPPT (Maximum Power Point Tracker) контроллерами, что позволяет повысить энергоотдачу ПИ до $30 \%$ [4].

Современная электронная аппаратура, питаемая от автономных СЭП, имеет широкий номенклатурный состав и зачастую импульсный характер потребления электроэнергии. Электропитание такой аппаратуры должно осуществляться стабильным напряжением с малыми допустимыми отклонениями напряжения в статических и динамических режимах работы [5]. Следовательно, кроме функций по передаче энергии и экстремальному регулированию ПИ, импульсный преобразователь ЭПА должен обеспечивать стабильность напряжения на выходных шинах СЭП, т. е. должен работать в режиме импульсного стабилизатора напряжения [6, 7]. Малые отклонения напряжения на выходных шинах СЭП обеспечиваются за счёт работы импульсных преобразователей в режиме импульсного стабилизатора напряжения (ИСН), в устройстве управления которого применены высокоэффективные законы управления [8-10]. Такие законы реализуются, как правило, посредством ШИМ-контроллеров, выпускаемых промышленностью в виде специализированных аналоговых микросхем [11-13].

Помимо задачи стабилизации выходного напряжения ШИМ-контроллеры обеспечивают ряд сервисных функций, например защиту стабилизаторов от перегрузки по току, от выхода входного напряжения за установленные пределы и превышения температурного режима работы $[14,15]$.

\section{Постановка задачи}

Для энергоэффективного производства и преобразования энергии возобновляемых источников импульсные преобразователи энергии, входящие в состав СЭП, должны иметь возможность обеспечивать как режим генерации максимальной мощности ПИ, так и режим стабилизации напряжения на выходных шинах. При отборе максимальной мощности от ПИ устройство управления импульсным преобразователем - МРРТ контроллером - обеспечивает перевод и удержание рабочей точки в окрестности максимума мощности на мощностной характеристике ПИ. В таком устройстве управления реализуется достаточно сложный алгоритм экстремального регулирования и на современном уровне развития техники устройство управления выполняется на основе программируемого цифрового устройства. Применение этого же программируемого цифрового устройства одновременно и для цели управления импульсным преобразователем в режиме стабилизации напряжения на выходных шинах является актуальной задачей. Это позволит отказаться от аналоговых ШИМ-контроллеров и тем самым уменьшить количество элементов в устройстве управления преобразователем, что снизит собственное энергопотребление и масса-габаритные характеристики, а также повысит надёжность функционирования импульсных преобразователей и СЭП в целом.

Наиболее целесообразным и соответствующим современным подходам следует считать реализацию устройства управления импульсным преобразователем на основе микропроцессорной техники со статически и динамически реконфигурируемой архитектурой. Такие устройства могут быть изготовлены в виде "систем на кристалле» (System-onChip, SoC) или реализованы на основе частично или полностью программируемых логических интегральных схемах со встроенным микропроцессорным ядром (Application-specific standard product/Field-Programmable Gate Array, ASSP/FPGA). Использование реконфигурируемой микропроцессорной техники позволит не только реализовать сложные алгоритмы экстремального регулирования мощности ПИ и новые законы управления преобразователем, работающим в режиме ИСН, но и дополнительные функции по защите от перегрузок, самодиагностике и телеметрии импульсных преобразователей [16, 17].

Из известных методов синтеза законов управления, обеспечивающих ИСН высокое быстродействие при стабилизации выходного напряжения, особый интерес представляет метод [18], заключающийся:

- в представлении силовых цепей стабилизаторов с ШИМ в режиме малых отклонений адекватной амплитудно-импульсной моделью по регулируемым составляющим процессов;

- синтезе закона управления с использованием полиномиальных уравнений синтеза систем с 
амплитудно-импульсной модуляцией (АИМ) [19];

- реализации синтезированного закона управления в ИСН с учетом специфики ШИМ.

Этот метод позволил авторам синтезировать закон управления, обеспечивающий минимальную конечную длительность переходных процессов в ИСН с силовой цепью понижающего типа, приведенной на рис. 1.

\section{Синтезированный закон управления}

Синтезированный закон управления ИСН назван авторами оптимальным по быстродействию, а ИСН с таким законом управления - быстродействующим ИСН.

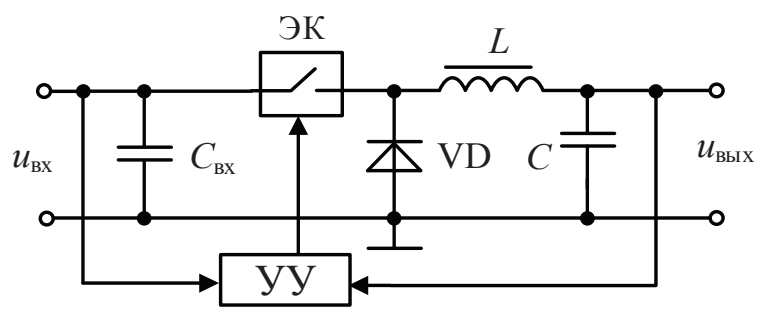

Pис. 1. Функциональная схема ИСН с силовой цепью понижающего типа

Fig. 1. Function chart of switching voltage regulator (SVR) with a power chain of the lowering type

В данном случае под регулируемыми составляющими процесса понимают отклонения переменных ИСН от их значений в стационарном режиме, обусловленные приращением текущей дли- тельности импульса $t_{\text {н.р }}$ относительно стационарной длительности $t_{\text {и.сг }}$.

Рассмотрим выделение регулируемой составляющей на примере временных диаграмм процессов в ИСН с силовой цепью понижающего типа, приведенных на рис. 2. На временных диаграммах (рис. 2) показаны: ток нагрузки $I_{\text {н }}$, ток дросселя $I_{L}$ и его стационарная составляющая $I_{L, c r}$, ток выходного конденсатора $I_{C}$ и его стационарная $I_{\text {С.ст }}$ и регулируемая $I_{C . p}$ составляющие, регулируемая составляющая $U_{C . \mathrm{p}}$ напряжения на выходном конденсаторе, период $T$ преобразования, длительность $t_{\text {и }}$ импульса и ее стационарная $t_{\text {и.ст }}$ и регулируемая $t_{\text {н.р }}$ составляющие, пилообразное напряжение широтно-импульсного модулятора $U_{\text {глин }}$.

Из общего процесса в ИСН с ШИМ вычленим стационарный процесс, соответствующий неизменной (стационарной) длительности $t_{\text {ист }}$ импульсов управления регулирующим элементом (РЭ), и процесс регулирования, обусловленный приращением длительности $t_{\text {и }}$ импульса управления на величину $t_{\text {н.р }}$ относительно стационарной длительности $t_{\text {н.ст }}$. Полезную информацию о процессе регулирования несут только регулируемые составляющие. Поэтому для процесса регулирования в режиме малых отклонений, когда $t_{\text {и.р }}<<T$, где $T-$ период преобразования, переход от системы с ШИМ к системе с АИМ осуществим путем замены регулируемой составляющей импульсов напряжения $u_{L p}(t)$, воздействующих со стороны РЭ на дроссель и имеющих длительность $t_{\text {н.р }}(t)$, эквивалентными по вольт-секундной «площади» $\delta$-функциями. В [20] показано, что при оптимальном по быстро-

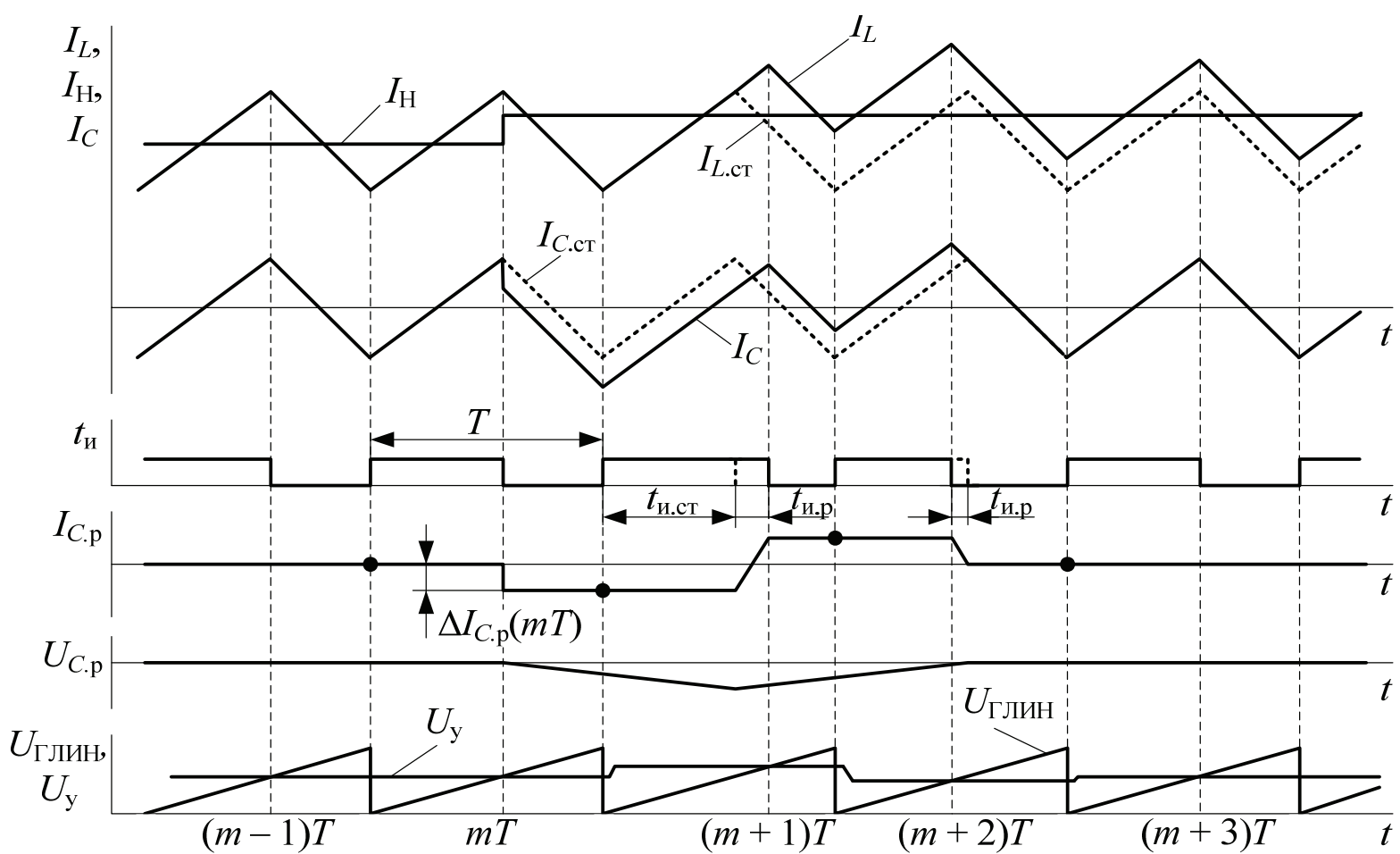

Рис. 2. Временные диаграммы процессов в ИСН с силовой цепью понижающего типа

Fig. 2. Diagram of processes of SVR with a power chain of the lowering type 
действию управлении в АИМ модели ИСН можно пренебречь влиянием проводимости нагрузки и внутреннего активного сопротивления дросселя. Это позволяет представить амплитудно-импульсную модель ИСН в виде последовательно включенных идеального импульсного элемента и звеньев с передаточными функциями $1 / p L$ и $1 / p C$ (рис. 3 ).

Регулируемая составляющая напряжения $u_{L . p}(t)$ на входе фильтра ИСН представляет двухполярные импульсы с амплитудой $u_{L \text { р.а }}(t)$ и длительностью $t_{\text {н.р }}(t)$, равной отклонению текущей длительности импульса $t_{\text {и }}(t)$ от стационарного значе-

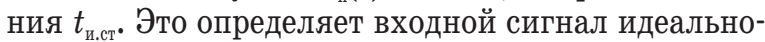
го импульсного элемента $s(t)=u_{\text {L.р.а }}(t) t_{\text {н.р }}(t)$ амплитудно-импульсной модели ИСН для регулируемых составляющих. Для этой модели

$$
u_{L . \mathrm{p}}(t)=s(t) \delta^{*}(t)
$$

где $\delta^{*}(t)=\sum_{m=1}^{\infty} \delta(t-m T)$ - последовательность $\delta$-функций с периодом $T$.

Процесс ШИМ-регулирования для регулируемых составляющих в области изображений определяется уравнением:

$$
U_{\text {вых }}^{*}(p)=W_{0}^{*}(p) S^{*}(p),
$$

где $U_{\text {вых }}^{*}(p)$ и $S(p)$ - изображения решетчатых функций регулируемых составляющих выходного напряжения и сигнала управления соответственно; $W_{0}^{*}(p)$ - дискретная передаточная функция, определяемая по передаточной функции непрерывной части $W_{\text {нч}}(p)[18]$.

Полезную информацию о процессе широтноимпульсного регулирования несут только регулируемые составляющие переменных состояния. Постоянная составляющая такой информации не содержит, а пульсация переменных состояний представляет информационную помеху. Это означает, что синтез законов управления ИСН необходимо осуществлять на основании математического описания процесса широтно-импульсного регулирования (2) с учётом (1), а влияние пульсации переменных состояний, в функции которых определен закон управления, учитывать при его реализации.

Приведение широтно-импульсного регулирования в окрестности стационарного режима к амплитудно-импульсному позволяет применить для анализа и синтеза ИСН с ШИМ хорошо разработанный аппарат теории систем с АИМ [18] и получить на его основе импульсные законы управления.
Структурная схема синтезируемой импульсной системы приведена на рис. 3.

Для синтеза оптимального по быстродействию закона управления ИСН используется третье полиномиальное уравнение синтеза [19]. Использование этого уравнения обеспечивает синтез дискретной передаточной функции $W_{\text {к }}(p)$ последовательного корректирующего устройства (ПКУ) из условия минимальной конечной длительности процессов в замкнутой системе по внешним воздействиям и при отклонении параметров корректирующего устройства от расчетных, что обусловливает практическую реализуемость $W_{\mathrm{k}}(p)$.

$$
W_{\mathrm{\kappa} 1}^{*}(p)=W_{\mathrm{\kappa} 2}^{*}(p)=d_{0}+d_{1}\left(1-e^{-p T}\right),
$$

где $d_{0}=d_{1}=\frac{1}{T \omega_{\phi}^{2}}, \omega_{\phi}^{2}=\frac{1}{L C}, L$ и $C-$ индуктивность и емкость выходного фильтра.

\section{Разработанные способы управления стабилизаторами}

В соответствии с синтезированной передаточной функцией последовательного корректирующего устройства (3) при реализации ПКУ для ИСН с ШИМ с учетом специфики, вносимой ШИМ, получены два варианта [18]. Первый, по мгновенным значениям переменных состояния, предполагает предварительное изучение влияния стационарной пульсации на процесс формирования модулятором приращений длительности импульсов управления $t_{\text {н.р }}(t)$ с последующим учетом этого влияния [20]. По данному варианту реализации ПКУ в ИСН с ШИМ проведены исследования, подтвердившие достижение в ИСН длительности переходных процессов в 3-4 периода преобразования, что близко к теоретическому пределу в два периода для системы второго порядка с амплитудно-импульсной модуляцией [20]. В то же время выявлена значительная статическая ошибка стабилизации выходного напряжения ИСН, обусловленная тем, что коэффициент усиления $d_{0}=d_{1}=1 /\left(T \omega_{\phi}^{2}\right)$ контура обратной связи, определяемый выражением (3), получен через параметры силовой цепи ИСН и не может быть увеличен без потери быстродействия [20]. В ИСН с первым вариантом управления по мгновенным значениям переменных состояния задача обеспечения астатизма выходного напряжения решена за счет введения второго интегрирующего контура обратной связи по напряжению [21]. При этом на вход широтно-импульсного модулятора поступает сигнал:

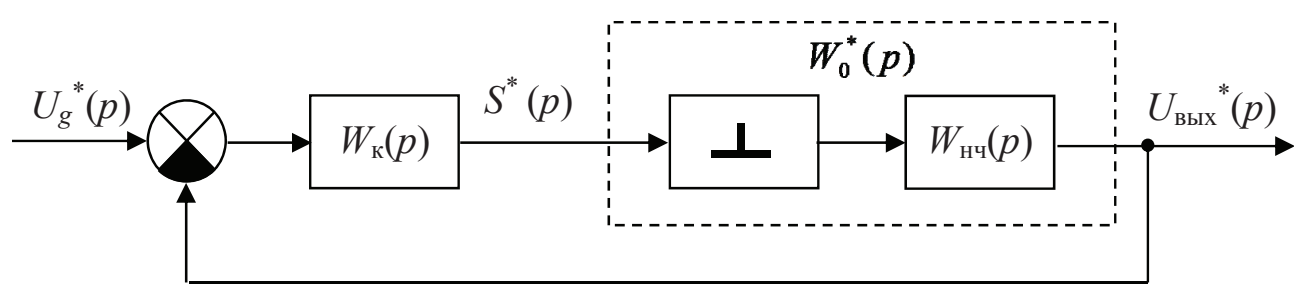

Рис. 3. Структурная схема синтезируемой импульсной системы

Fig. 3. Block-diagram of the synthesized pulse system 


$$
u_{\text {вX.M }}(t)=u_{\text {д }}(t)+u_{\text {инт }}(t)
$$

в виде суммы двух сигналов, первый из которых $u_{\text {д }}(t)$, формируемый последовательным корректирующим устройством, разработанным на основе (3), обеспечивает динамические свойства ИСН, а второй $-u_{\text {инт }}(t)$, обеспечивает астатизм выходного напряжения ИСН. Сигнал $u_{\text {инт }}(t)$ получен интегрированием сигнала рассогласования $e(t)$ по выходному напряжению ИСН:

$$
u_{\text {инт }}(t)=K_{\mathrm{p}} \int_{0}^{t} e(t) d t+U_{1},
$$

где $e(t)=u_{\text {вых }}(t)-U_{0}, u_{\text {вых }}(t)$ - напряжение на выходе ИСН, a $U_{0}$ - эталонное напряжение. Коэффициент $K_{\mathrm{p}}<<4 K_{\text {опт }} /\left(2 r_{c} C+T\right)$, где $K_{\text {опт }}-$ коэффициент усиления в контуре обратной связи, определяющем динамические свойства ИСН, $r_{C}$ - внутреннее активное сопротивление конденсатора выходного фильтра; $C$ - емкость конденсатора выходного фильтра.

Второй способ реализации основан на выделении регулируемых составляющих переменных состояния стабилизатора специальными измерительными устройствами - устройствами выборки и хранения в начале периода преобразования. При реализации устройства управления ИСН на основе микропроцессорной техники второй способ предпочтительней первого, поскольку предполагает однократную выборку сигналов на периоде преобразования, в то время как реализация устройства управления по первому способу потребовала бы многократное оцифровывание информационных сигналов. Согласно второму варианту реализации ПКУ [21], входной сигнал широтно-импульсного модулятора, определяющий динамические свойства ИСН, формируется как:

$$
U_{\text {д }}(m T)=U_{\text {д }}((m-1) T)+\Delta U_{\text {д }}(m T),
$$

а его приращения за период $T$ :

$$
\begin{gathered}
\Delta U_{\text {д }}(m T)= \\
=\frac{L C}{U_{\text {вх }} K_{\mathrm{M}} T^{2}}\left[2 \Delta U_{C . \mathrm{p}}(m T)-\Delta U_{C . \mathrm{p}}((m-1) T)\right],
\end{gathered}
$$

где $U_{\text {вх }}$ - напряжение на входе ИСН, первая разность напряжения на выходе ИСН:

$$
\Delta U_{C . \mathrm{p}}(m T)=U_{C . \mathrm{p}}(m T)-U_{C . \mathrm{p}}((m-1) T),
$$

коэффициент передачи широтно-импульсного модулятора:

$$
K_{\text {м }}=\Delta t_{\text {и.у }} / \Delta U_{\text {вх.м }}(m T)=T / U_{m},
$$

$\Delta t_{\text {н.у }}$ - приращение длительности импульса управления силовым ключом ИСН, $\Delta U_{\text {вх.м }}(m T)$ - приращение входного сигнала широтно-импульсного модулятора; $U_{m}$ - амплитуда пилообразного напряжения ШИМ [21].

В настоящее время прогресс в области микропроцессорной техники позволяет решать задачи построения устройства управления ИСН с использованием второго варианта реализации. При этом возможно создать ИСН с оптимальным по быстро- действию законом управления при использовании интегрирующего контура обратной связи по напряжению, функционирующего под управлением цифрового ШИМ.

\section{Решение задачи минимизации переходных процессов}

Решение задачи минимизации переходных процессов в данном случае сводится к получению средствами микропроцессорной техники входного сигнала ШИМ с использованием выражения, аналогичного (4), и формированию посредством этого сигнала собственно широтно-модулированного импульсного сигнала управления силовым ключом ИСН.

Входной сигнал ШИМ определим по выражению, аналогичному (4), которое учитывает дискретный характер обработки сигналов:

$$
U_{\mathrm{BX} . \mathrm{M}}(m T)=U_{\text {д }}(m T)+U_{\text {инт }}(m T) \text {. }
$$

Сигнал $U_{\text {л }}(m T)$, определяющий динамические свойства ИСН, вычисляется по выражениям (6)-(8). Причем первая разность регулируемой составляющей напряжения на выходе ИСН, определяемая по выражению (8), может быть найдена по выражению:

$$
\Delta U_{C . \mathrm{p}}(m T)=E(m T)-E((m-1) T),
$$

где $E(m T)$ - дискретное значение сигнала рассогласования по выходному напряжению ИСН, определяемое аналогично (5) по выражению:

$$
E(m T)=U_{\text {вых }}(m T)-U_{0} .
$$

Такая замена возможна, поскольку напряжение на выходе ИСН

$$
U_{\text {вых }}(m T)=U_{C . \mathrm{p}}(m T)+U_{\text {пост }}(m T)+U_{\text {пульс }}(m T),
$$

кроме регулируемой составляющей $U_{c . p}(m T)$, содержит постоянную составляющую $U_{\text {пост }}(m T)$ и пульсацию $U_{\text {пульс }}(m T)$, которые не изменяются за период $T$. Следовательно при вычислении первой разности $\Delta U_{C . \mathrm{p}}(m T)$ по выражению (11), с учетом (12), постоянная составляющая $U_{\text {пост }}(m T)$, пульсация $U_{\text {пульс }}(m T)$ выходного напряжения и эталонное напряжение $U_{0}$, смещенные на период $T$ друг относительно друга, взаимно вычитаются. Сигнал $U_{\text {инт }}(m T)$, обеспечивающий астатизм ИСН, может быть вычислен по выражению:

$$
U_{\text {инт }}(m T)=K_{\mathrm{p}} \sum_{k=1}^{m} E(k T),
$$

аналогичному (5), с учетом замены суммированием операции интегрирования.

Выборка необходимых для управления информационных сигналов, а именно напряжений на входе и выходе ИСН, производится в моменты времени $m T$. Процедура оцифровывания сигналов и последующих математических операций занимает некоторый интервал времени $t_{\text {см }}<<$. В рассматриваемом случае осуществляется модуляция переднего фронта импульса управления силовым ключом ИСН и задержка в определении длительности импульса управления силовым ключом ИСН не оказывает существенного влияния на результат вычислений, поскольку $t_{\text {см }}<<T$ и вычислительные 


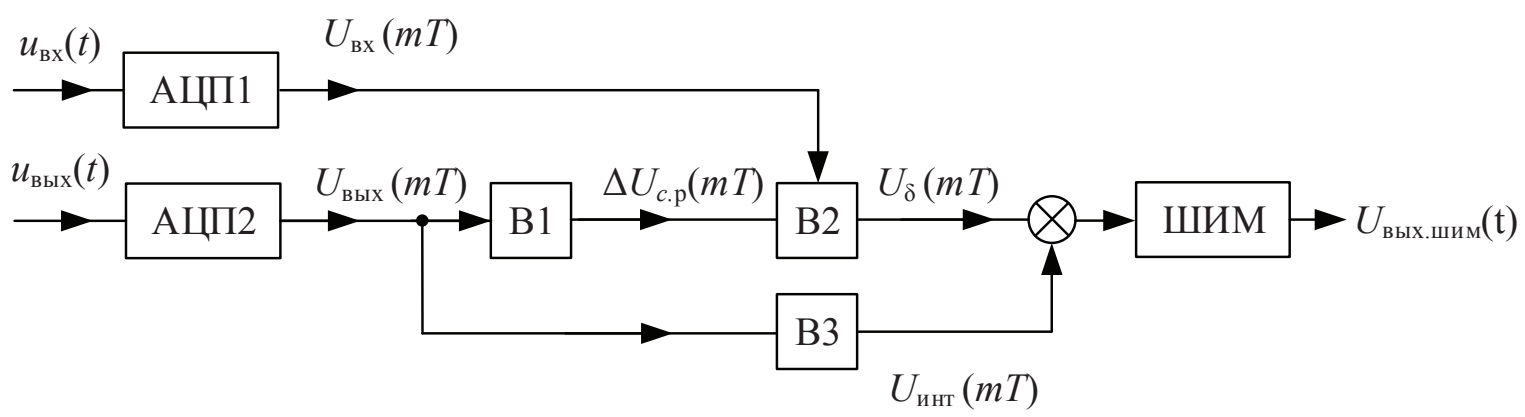

Рис. 4. Схема последовательности обработки сигналов устройством управления

Fig. 4. Scheme of sequence in signal processing by a control unit

операции производятся на интервале выключенного состояния силового ключа.

На рис. 4 приведена структурная схема последовательности обработки сигналов микропроцессорной системой.

В моменты времени $m T$ аналого-цифровые преобразователи АЦП1 и АЦП2 обеспечивают выборку информационных сигналов, соответственно входного $u_{\text {вх }}(t)$ и выходного $u_{\text {вых }}(t)$ напряжений ИСН, и последующую оцифровку выбранных значений сигналов, т. е. определение сигналов $U_{\text {вх }}(m T)$ и $U_{\text {вых }}(m T)$. Далее в окрестности момента времени $m T$ вычислитель B1, в соответствии с (11), выполняет расчет первой разности $\Delta U_{C . p}(m T)$ регулируемой составляющей напряжения на выходе ИСН. Затем вычислитель B2 формирует сигнал $U_{\text {д }}(m T)$, определяющий динамические свойства ИСН, а вычислитель В3 - сигнал $U_{\text {инт }}(m T)$, обеспечивающий астатизм выходного на- пряжения ИСН. Далее сумматор С1, согласно (10), складывая сигналы $U_{\text {д }}(m T)$ и $U_{\text {инт }}(m T)$, вычисляет $U_{\text {вх.м }}(m T)$, а затем ШИМ по выражению: $t_{\text {н.у }}=K_{\mathrm{M}} U_{\text {вх.м. }}$, полученному путем преобразования (9), вычисляет длительность импульса управления $t_{\text {и.у }}$ силовым ключом ИСН на периоде его работы, следующем за моментом времени $m T$. Микропроцессорная система синхронизирует задний фронт этого импульса управления с моментом времени $(m+1) T$.

\section{Практическая реализация синтезированного} закона и алгоритмов

С целью подтверждения полученных теоретических результатов и в соответствии с функциональной схемой, приведённой на рис. 1, авторами разработан и изготовлен макет ИСН понижающего типа с цифровым управлением. Функциональная схема макета ИСН приведена на рис. 5.

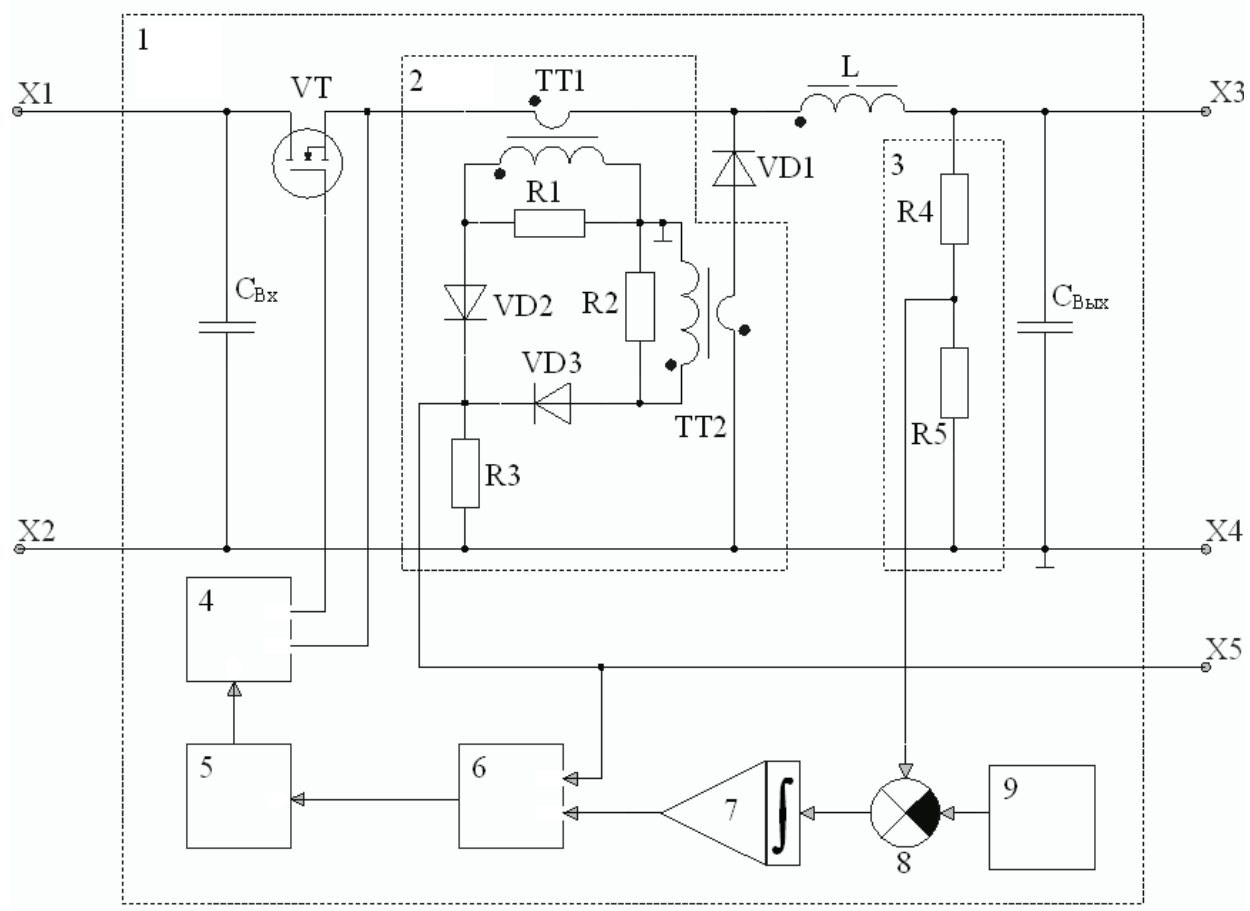

Pис. 5. Функциональная схема макета ИСН

Fig. 5. Functional diagram of SVR 
В состав силовой части макета - 1 входят: силовой ключ, выполненный на полевом транзисторе VT, замыкающий диод VD1, конденсаторы входного $C_{\text {вх }}$ и выходного $C_{\text {вых }}$ рильтров, дроссель $L$ и датчик тока дросселя - 2, выполненный с использованием трансформаторов тока ТT1 и ТТ2 с резисторами размагничивания $\mathrm{R} 1$ и $\mathrm{R} 2$, диодов VD2, VD3 и информационного резистора R3. B состав устройства управления макета входят: делитель напряжения - 3 , состоящий из резисторов R4 и $\mathrm{R} 5$, драйвер - 4, формирующий напряжение на затворе транзистора VT, управляющий автомат - 5 на базе ПЛИС Altera Cyclone III EP3C120F780 FPGA со встроенным процессорным ядром NIOS-II, аналогоцифровой преобразователь - 6 на базе max1308, аналоговый интегратор - 7 и сумматор 8 , выполненные на основе операционного усилителя, и источник опорного напряжения -9 .

Изготовленный макет ИСН имеет следующие основные характеристики: напряжение на выходе ИСН $u_{\text {вых }}=28 \mathrm{~B}$, напряжение на входе $u_{\text {вх }}$ от 40 до $110 \mathrm{~B}$, частота преобразования $f_{\text {пр }}=120$ кГц, ёмкость конденсаторов входного $C_{\text {вх }}$ и выходного $C_{\text {вых }}$ фильтров составляет 1000 мкФ, индуктивность дросселя $L-180$ мкГн при токе в 1,5 А и 120 мкГн при токе в $4 \mathrm{~A}$.

Макет предназначен для определения динамических характеристик, близких к предельно возможным, в сочетании с астатизмом выходного напряжения в статических режимах работы.

Реализованное устройство управления на микропрограммном уровне использует алгоритм формирования сигнала управления силовым ключом ИСН, приведённый на рис. 6. При этом в расчётных соотношениях принято среднее значение величины индуктивности дросселя в 150 мкГн.

Разработано встроенное программное обеспечение, которое функционирует следующим образом. Изначально, с целью завершения переходных процессов в цифровых и аналоговых модулях системы, а также выхода электронных компонент источника на рабочий режим, вводится программная задержка стартового замера, в процессе которой происходит инициализация начальных значений переменных, настройка подсистем управления и определение системных констант. Здесь и далее интервальные линии реализуются на аппаратнопрограммном уровне при помощи программируемых таймеров-счетчиков.

Результатом функционирования встроенного программного модуля будет формирование управляющего импульса с предварительным расчетом его корректирующих значений $-\Delta U_{C . \text { рi }}$ и $U_{\text {упрi }}$. Изначально эти переменные должны быть «сброшены» - приравнены к заданному коэффициенту пересчета.

На первоначальном этапе замера, обозначим его как этап $(i-1)$, происходит оцифровка входных сигналов посредством АЦП 1 и 2 (рис. 4). При этом драйверы АЦП 1 и 2 выполняют снятие и представление в числовом формате значений $U_{\text {вх }}(i-1)$,
$U_{\text {вых }}(i-1)$ соответственно. Эти АЦП функционируют параллельно, и начало преобразования - синхронно. Тем не менее возможно некоторое рассогласование завершения этапов преобразования. Здесь интервалы оцифровки могут иметь предельно малую разницу, порядка $10^{-12} \mathrm{c}$, что не имеет принципиального недостатка во временных интервалах расчета и синхронизируется при приеме данных микропроцессорным ядром от АЦП в режиме прямого доступа к памяти. АЦП выполняют преобразование в течение малого интервала смещения $\tau<0,25 T$, т. е. в течение первой четверти периода.

По окончании замеров вычислитель В3 производит расчет значения сигнала рассогласования по напряжению согласно выражению (12)

$$
E(i):=U_{\text {вых }}(i-1)-U_{0} \text {. }
$$

На следующем этапе расчетов, в потоковом режиме, функционируют вычислители В1 и В2, производящие вычисление приращения текущей регулируемой составляющей напряжения на емкости конденсатора $\Delta U_{C . \mathrm{p}}(i)=E(i)-E(i-1)$, а также текущего приращения входного сигнала широтноимпульсного модулятора $U_{\text {д }}(i)=U_{\text {д }}(i-1)+\Delta U_{\text {д }}(i)$ соответственно, согласно выражениям (11) и (6). Полученные результаты занимают место предыдущих в памяти системы, т. е. вновь выполняется сдвиг массива значений. Посредством вычислителя В3, в соответствие с выражением (13), записанным для интервала $i$, вычисляют $U_{\text {инт }}(i)$. Далее, в соответствии с выражением $(10) U_{\text {вх.м }}(i)=U_{\text {д }}(i)+U_{\text {инт }}(i)$, вычисляется сигнал $U_{\text {вх.м }}(i)$, и по выражению $t_{\text {н.у }}(i)=K_{\mathrm{m}} U_{\text {вх.м }}(i)$ определяется длительность сигнала управления силовым ключом ИСН. Полученные результаты переписываются в память со сдвигом значений, и цикл повторяется.

Алгоритм функционирования разработанного встроенного программного обеспечения приведен на рис. 6.

\section{Результаты тестирования макета}

Для проведения исследований использовалась экспериментальная установка, функциональная схема которой приведена на рис. 7.

В состав экспериментальной установки входят: макет ИСН - 1, источник питания - 2 с диапазоном регулирования питающего напряжения от 40 до $110 \mathrm{~B}$, датчик тока - 3 в виде резистивного шунта $\mathrm{R}_{\text {ш }}$ сопротивлением в 0,2 Ом, блок нагрузок -4 в виде постоянно подключенного и периодически коммутируемого резисторов, и четырёхканальный осциллограф - 5 .

Полученные результаты исследования изготовленного макета ИСН приведены на рис. 8-10. На всех осциллограммах луч 1 (по порядку сверху вниз) показывает изменение тока нагрузки в масштабе 2,5 А/дел, луч 2 - ток дросселя в масштабе $2 \mathrm{~A} /$ дел и луч 3 - переменную составляющую напряжения на выходе ИСН в масштабе 0,2 В/дел. Постоянная составляющая тока нагрузки на осциллограммах показана частично. На осциллограммах, приведенных на рис. 8, показаны процессы, 


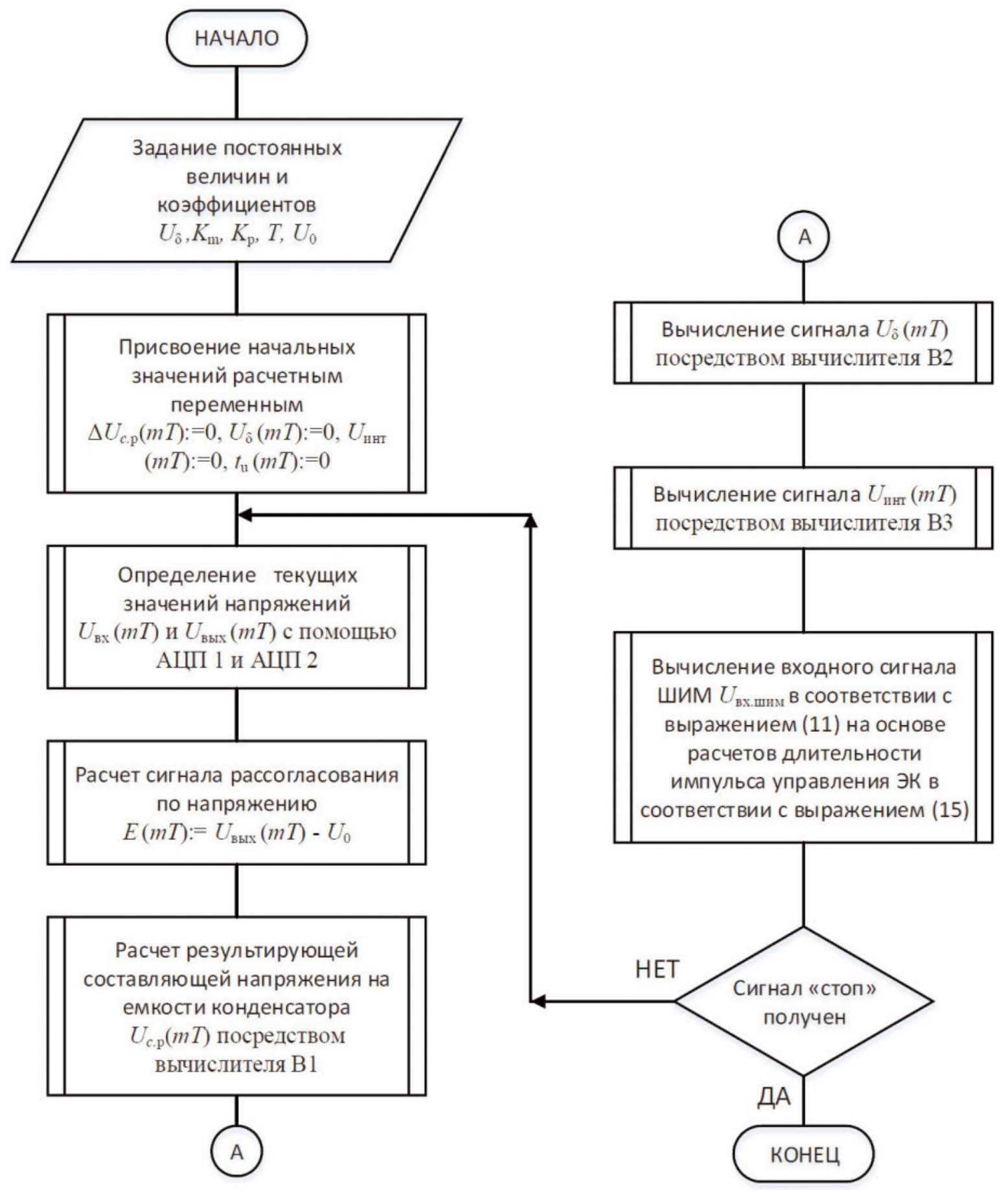

Рис. 6. Алгоритм функционирования встроенного программного обеспечения ИСН

Fig. 6. General algorithm of embedded software of SVR

включающие два периода ступенчатого изменения тока нагрузки. Постоянная составляющая тока нагрузки равна $1,4 \mathrm{~A}$, а ступенчатое приращение тока нагрузки составляет 2,8 А. Из осциллограммы 3 видно, что по окончании переходного процесса выходное напряжение возвращается к исходному значению, т. е. отсутствует статическая ошибка стабилизации напряжения. Дополнительные ис- следования подтвердили отсутствие статической ошибки стабилизации напряжения и при вариации входного напряжения ИСН в указанном выше диапазоне. На рис. 9, 10 укрупненно показаны процессы, соответственно, в окрестности ступенчатого уменьшения и увеличения тока нагрузки. При ступенчатом уменьшении тока нагрузки (рис. 9) длительность переходного процесса от момента изме- 


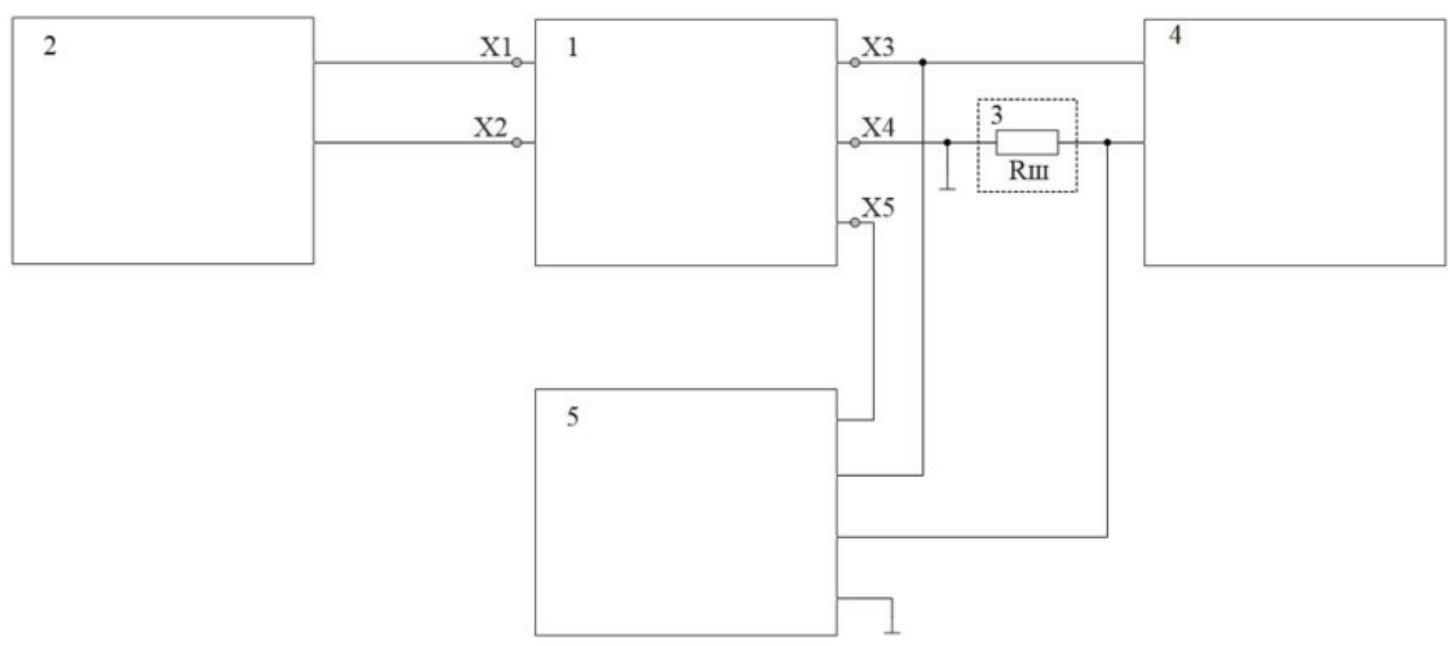

Рис. 7. Функциональная схема экспериментальной установки

Fig. 7. Functional diagram of the trial type

нения тока нагрузки до момента наступления установившегося периодического режима изменения тока дросселя и напряжения на выходе ИСН составляет 3-4 периода, что близко к минимально возможной длительности переходного процесса. При ступенчатом увеличении тока нагрузки (рис. 10) длительность переходного процесса составляет 6-8 периодов. Такое увеличение длительности переходного процесса относительно минимально возможной объясняется установленным ограничением максимальной длительности импульса управления силовым ключом ИСН в 0,75 Т. Это вызвано, во-первых, необходимостью введения временного интервала для размагничивания трансформатора TT1 датчика тока дросселя, и, во-вторых, для проведения процедур оцифровывания информацион- ных сигналов и вычисления длительности импульса управления силовым ключом ИСН.

\section{Заключение}

Исследование процессов в макете импульсного стабилизатора напряжения с предложенным устройством управления показало его эффективное функционирование. Так, при ступенчатом изменении тока нагрузки достигается малая длительность переходного процесса изменения выходного напряжения, составляющая три-пять периодов преобразования. Показано, что необходимо и достаточно за период преобразования выполнить однократно процедуры оцифровывания и вычисления. При этом освобождается существенный временной интервал в работе цифрового вычислитель-

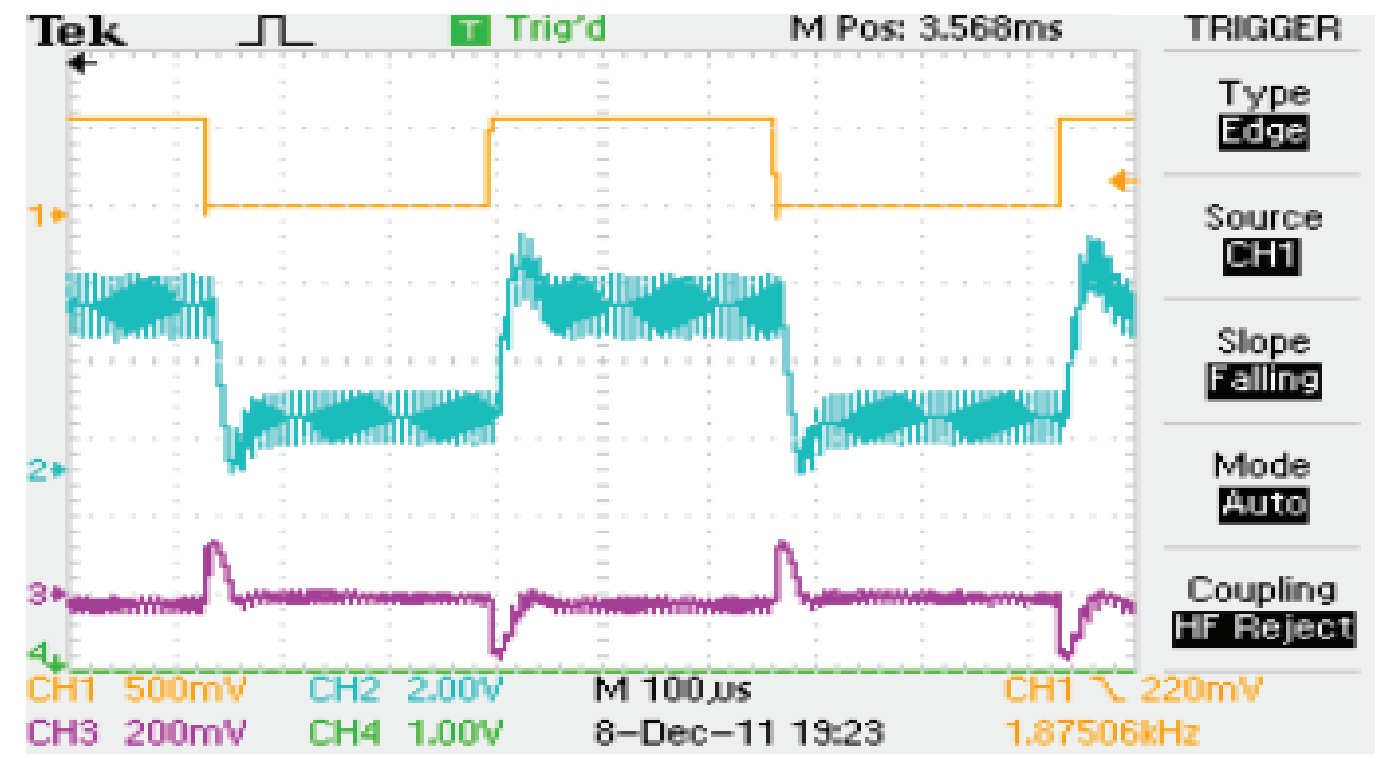

Рис. 8. Ступенчатое изменение тока нагрузки

Fig. 8. Step change of output current 


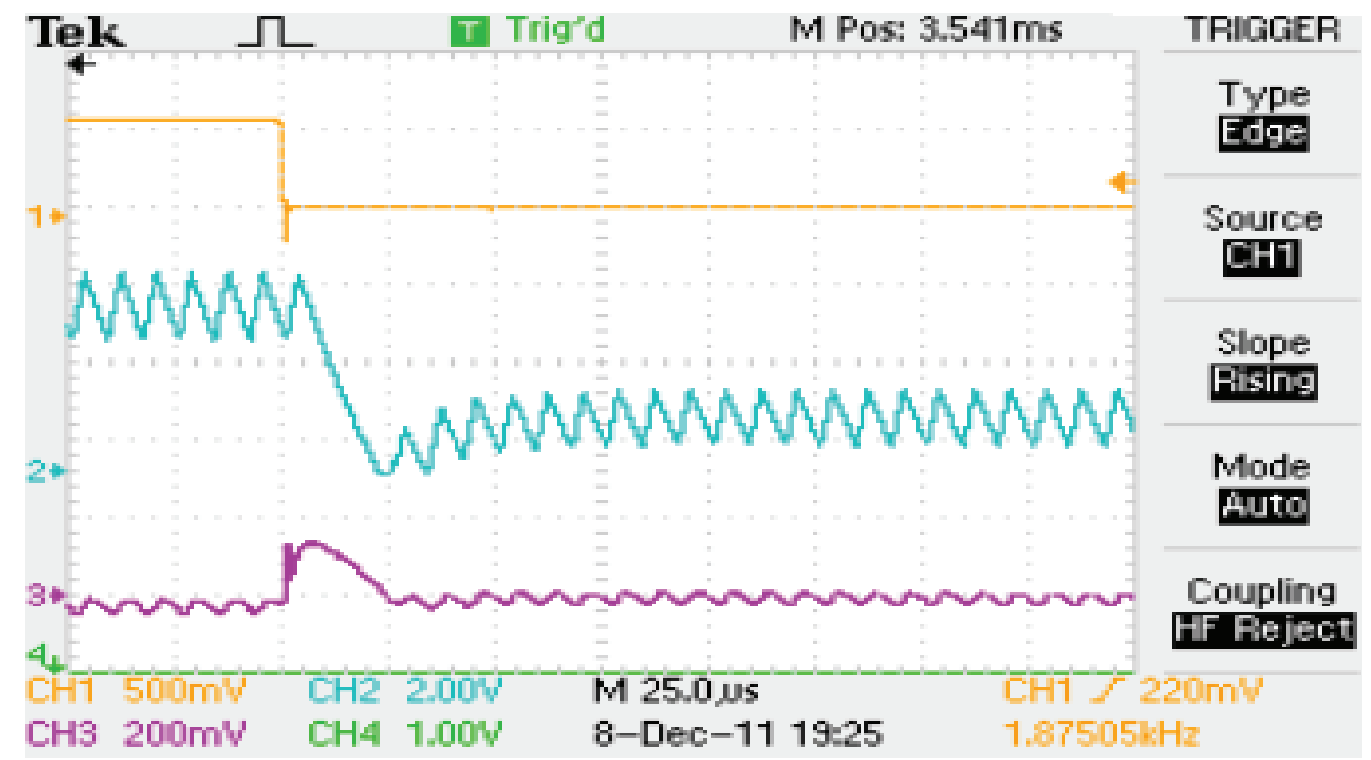

Рис. 9. Ступенчатое уменьшение тока нагрузки

Fig. 9. Step reduction of output current

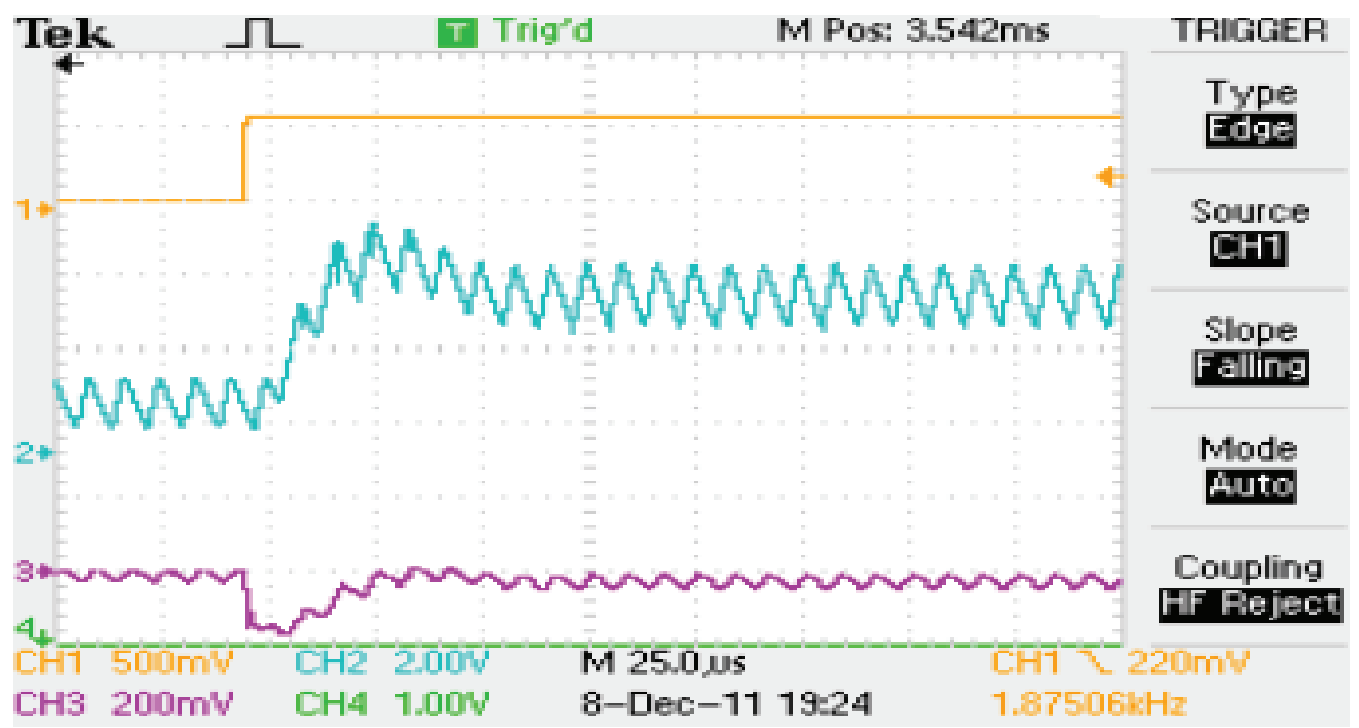

Рис. 10. Ступенчатое увеличение тока нагрузки

Fig. 10. Step increase of output current

ного устройства, который может быть использован, например, для решения задач по экстремальному регулированию мощности первичного источника, ограничению выходного тока на заданном уровне и по распределению тока нагрузки между отдельными ИСН при их параллельной работе на общую нагрузку, диагностике функционирования ИСН, что является дополнительным преимуще- ством предложенного способа управления. Таким образом, импульсный стабилизатор напряжения с полностью цифровым контуром управления, реализованным при помощи высокоскоростных микропроцессорных средств, имеет значительные преимущества по сравнению с аналоговыми вариантами и обладает неоспоримыми преимуществами стратегического плана. 


\section{СПИСОК ЛИТЕРАТУРЫ}

1. Ellabban Omar, Abu-Rub Haitham, Blaabjerg Frede. Renewable energy resources: current status, future prospects and their enabling technology // Renewable and Sustainable Energy Reviews. - 2014. - V. 39. - № C. - P. 748-764.

2. Русскин В.А., Семёнов С.М., Диксон Р.К. Исследование алгоритмов поиска точки максимальной мощности для повышающего преобразователя напряжения солнечного инвертора // Известия Томского политехнического университета. Инжиниринг георесурсов. - 2016. - Т. 327. - № 4. - С. 78-87.

3. Вольтодобавочный последовательный резонансный преобразователь с изменяемой структурой для систем электропитания / А.В. Осипов, Е.В. Ярославцев, Е.Ю. Буркин, В.В. Свиридов // Известия Томского политехнического университета. Инжиниринг георесурсов. - 2018. - Т. 329. - № 2. - С. 27-37.

4. Имитационная модель контроллера солнечной батареи / С.С. Пост, О.А. Донцов, В.И. Иванчура, Ю.В. Краснобаев // Известия Томского политехнического университета. - 2014. T. 325. - № 4. - C. 111-120.

5. Краснобаев Ю.В., Пожаркова И.Н. Определение допустимых значений выходного импеданса автономной системы электропитания // Вестник Сибирского аэрокосмического университета. - 2007. - Вып. 3 (16). - С. 91-96.

6. Direct Buck Converter with Zero Voltage Transition and PWM Control (ZVT-PWM) / S.S. Tyunin, V.A. Kabirov, V.D. Semenov A.V. Kobzev $/ / 13^{\text {th }}$ International scientific-technical conference on actual problems of electronic instrument engineering (APEIE). - Novosibirsk, 2016. - V. 1. - № 3. - P. 29-35.

7. Direct Boost Converter with Zero Voltage Transition and PWM Control (ZVT-PWM)* / D.B. Borodin, V.A. Kabirov, N.P. Vintonyak, V.D. Semenov, Y.A. Shurygin $/ / 13^{\text {th }}$ International scientific-technical conference on actual problems of electronic instrument engineering (APEIE). - Novosibirsk, 2016. - V. 1. - № 3. P. 22-28.

8. Tanovitskiy Y., Kobzev G., Savin D. Minimizing the Duration of the Transient Processes in a Buck Converter with Pulse Width Modulation and Inert Output Filter // Procedia Technology. 2014. - № 18. - P. 6-13.

9. Minimum Time Control for Multiphase Buck Converter: Analysis and Application / P. Cheng, M. Vasic, 0. Garcia, J.A. Oliver, P. Alou, J.A. Cobos // IEEE Transactions on Power Electronics. - 2014. - № 29/2. - P. 958-967.
10. Modeling and Control of a New Three-Input DC-DC Boost Converter for Hybrid PV/FC/Battery Power System / F. Nejabatkhah, S. Danyali, S.H. Hosseini, M. Sabahi, S.M. Niapour // IEEE Transactions on Power Electronics. - 2012. - № 27/5. P. 2309-2324.

11. ШИМ-контроллеры Texas Instruments // Компэл. URL: https://www.compel.ru/lib/ne/2007/3/5-shim-kontrolleryi-texas-instruments (дата обращения: 11.02.2018).

12. Штрапенин Г. Новое поколение интегральных микросхем. National Semiconductor для импульсных источников питания // Компоненты и технологии. - 2007. - № 8. - С. 114-118.

13. Аналоговый мир Maxim. Новые микросхемы питания. - М.: Изд-во. Maxim Integrated Products, 2014. - № 3. - 60 c.

14. Wen Y., Trescasesr 0. DC-DC Converter with Digital Adaptive Slope Control in Auxiliary Phase for Optimal Transient Response and Improved Efficiency // IEEE Transactions on Power Electronics. - 2012. - № 27/7. - P. 3396-3409.

15. Zhou G., Xu J., Jin Y. Improved digital peak current predictive control for switching DC-DC converters // IET Power Electronics. - 2011. - № 4/2. - P. 227-234.

16. Винтоняк Н.П., Федотов В.А. Семенов В.Д. Методика проектирования программного обеспечения микроконтроллеров для быстродействующих систем управления // Доклады ТУСУРа. - 2015. - № 2 (36). - C. 175-179.

17. Digitally controlled multi-phase buck-converter with merged capacitive attenuator / B. Mahdavikhah, P. Jain, A. Prodic // Twenty-Seventh Annual IEEE Applied Power Electronics Conference and Exposition (APEC). - Orlando, 2012. - P. 1083-1087.

18. Системы электропитания космических аппаратов / Б.П. Соустин, В.И. Иванчура, А.И. Чернышев, Ш.Н. Исляев. - Новосибирск: В0 «Наука». Сибирская издательская фирма, 1994. $318 \mathrm{c.}$

19. Цыпкин Я.З. Теория линейных импульсных систем. - М.: Физматгиз, 1963. - 968 с.

20. Манаков А.В., Иванчура В.И., Соустин Б.П. Синтез и исследование быстродействующего импульсного стабилизатора напряжения с ШИМ // Техническая электродинамика. - 1987. № 1. - C. $43-51$.

21. Краснобаев Ю.В. Методология синтеза законов и структур устройств управления конверторами / Изв. вузов. Сер. Приборостроение. - 2004. - Т. 47. - Вып. 4. - С. 39-48.

Поступила 24.04.2018 г.

\section{Информация об авторах}

Краснобаев Ю.В., доктор технических наук, профессор, профессор кафедры систем автоматики, автоматизированного управления и проектирования Института космических и информационных технологий Сибирского федерального университета.

Непомнящий O.B., кандидат технических наук, доцент, профессор, заведующий кафедрой вычислительной техники Института космических и информационных технологий Сибирского федерального университета.

Иванчура В.И., доктор технических наук, профессор, профессор кафедры систем автоматики, автоматизированного управления и проектирования Института космических и информационных технологий Сибирского федерального университета.

Пожаркова И.Н., кандидат технических наук, доцент, доцент кафедры пожарно-технических экспертиз Сибирской пожарно-спасательной академии ГПС МЧС России; доцент кафедры систем автоматики, автоматизированного управления и проектирования Института космических и информационных технологий Сибирского федерального университета.

Яблонский А.П., магистрант кафедры вычислительной техники Института космических и информационных технологий Сибирского федерального университета. 
UDC 621.314 .2

\title{
PULSED VOLTAGE REGULATOR WITH DIGITAL CONTROL FOR AUTONOMOUS POWER SUPPLY SYSTEM
}

\author{
Yuriy V. Krasnobaev', \\ uvkras@mail.ru
}

Oleg V. Nepomnyashchiy',

2955005@gmail.com

\section{Vladimir I. Ivanchura', ivan43ura@yandex.ru}

\author{
Irina N. Pozharkova, ${ }^{2,}$, \\ pozharkova@mail.ru
}

\author{
Aleksey P. Yablonskiy', \\ lehateslo@gmail.com \\ 1 Siberian Federal University, \\ 26, Kirensky street, Krasnoyarsk, 660074, Russia. \\ 2 FSBEE HE Siberian Fire and Rescue Academy EMERCOM of Russia, \\ 1, Severnaya street, Zheleznogorsk, 662971, Russia.
}

Relevance. The efficiency and effectiveness of geophysical survey, geological work, meteorological observation and environmental monitoring depend on the power supply systems of testing and research equipment. Specificity of such works implies the use of field autonomous systems. Primary energy sources in such systems, as a rule, are renewable energy sources, such as solar batteries, wind or hydroelectric power plant, and secondary sources of energy are accumulator batteries. Energy sources are combined into a power supply system using pulse energy converters, which provide transferring energy and stabilizing supply voltage for consumers. An important function of pulse energy converters is increasing the energy efficiency of primary energy sources by means of their exploiting in the mode of generating maximum power. Consumers of autonomous power supply system are a complex and heterogeneous equipment, often having an impulse mode of energy consumption. This leads to significant voltage deviations on output buses of the power supply system and mutual influence of separate consumers and may cause malfunctions in their operation. Pulse energy converters must provide voltage stabilization on output buses. In the mode of maximum power generation, the pulse converter control unit provides conversion and holding of the operating point on the power characteristic of the primary source within the maximum power. The control unit implements a rather complicated algorithm of extreme control. At the modern level of technology development, the control unit is developed on the basis of a programmable digital device. The use of the same programmable digital control device for the mode of voltage stabilization on output buses is an actual task. This solution will reduce the complication of the converter control unit, decrease its own power consumption and improve operation reliability.

The aim of the work is to solve the theoretical and practical tasks of ensuring digital control with the pulse converter in stabilization mode of output tension ensuring small duration of transients caused by of loading current increment and output tension astatism.

Methods: theory of pulsed automatic control systems, mathematical modeling of processes in switching voltage regulators and physical prototyping.

Results. The authors have analyzed the features of autonomous power supply systems, synthesized the control law and developed the model of the pulse voltage stabilizer. The proposed method of the switching voltage regulator control provides the minimal transient duration. Microprogramming control algorithms are designed on the base of the specified control method. The authors developed the architecture of the microprocessor control system and built-in software and implemented a single-chip computing module of the control system using a programmable integrated circuit as well as a prototype of the switching voltage regulator with the digital control unit on the base of the single-chip computational module. A case study of the prototype shows the effectiveness of the developed control system. It is shown that the developed control system provides the minimum transient finite duration caused by a step change in the load current equal to two conversion periods. The test results confirmed that a fully digital control loop on the base of a high-speed microprocessor unit for switching voltage regulators has significant advantages over analog implementations.

\section{Key words:}

Renewables, autonomous power supply system, switching voltage regulator, digital control loop, embedded software, adjustable components of state variables.

\section{REFERENCES}

1. Ellabban Omar, Abu-Rub Haitham, Blaabjerg Frede. Renewable energy resources: current status, future prospects and their enabling technology. Renewable and Sustainable Energy Reviews, 2014, vol. 39, no. C, pp. 748-764.
2. Russkin V.A., Semenov S.M., Dixon R.C. Study of algorithms for tracking maximum power point for boost DC-DC converter of solar inverter. Bulletin of the Tomsk Polytechnic University. Geo Assets Engineering, 2016, vol. 327, no. 4, pp. 78-87.

3. Osipov A.V., Yaroslavtsev E.V., Burkin E.Yu., Sviridov V.V. Boost type series resonant converter with flexible structure for 
power supply. Bulletin of the Tomsk Polytechnic University. Geo Assets Engineering, 2018, vol. 329, no.2, pp. 27-37.

4. Post S.S., Dontsov 0.A., Ivanchura V.I., Krasnobaev Yu.V. Simulation model of a solar cell controller. Bulletin of the Tomsk Polytechnic University, 2014, vol. 325, no. 4, pp. 111-120.

5. Krasnobaev Yu.V., Pozharkova I.N. Determination of output impedance permissible values of an autonomous power supply system. Bulletin of the Siberian Aerospace University, 2007 vol. 3, pp. 91-96. In Rus.

6. Tyunin S.S., Kabirov V.A., Semenov V.D., Kobzev A.V. Direct Buck Converter with Zero Voltage Transition and PWM Control (ZVT-PWM). $13^{\text {th }}$ International scientific-technical conference on actual problems of electronic instrument engineering (APEIE). Novosibirsk, 2016. Vol. 1, no. 3, pp. 29-35.

7. Borodin D.B., Kabirov V.A., Vintonyak N.P., Semenov V.D., Shurygin Y.A. Direct Boost Converter with Zero Voltage Transition and PWM Control (ZVT-PWM)*. $13^{\text {th }}$ International scientific-technical conference on actual problems of electronic instrument engineering (APEIE). Novosibirsk, 2016. Vol. 1, no. 3, pp. 22-28.

8. Tanovitskiy Y., Kobzev G., Savin D. Minimizing the Duration of the Transient Processes in a Buck Converter with Pulse Width Modulation and Inert Output Filter. Procedia Technology, 2014, no.18, pp. 6-13.

9. Cheng P., Vasic M., Garcia 0., Oliver J.A., Alou P., Cobos J.A. Minimum Time Control for Multiphase Buck Converter: Analysis and Application. IEEE Transactions on Power Electronics, 2014, no. 29/2, pp. 958-967.

10. Nejabatkhah F., Danyali S., Hosseini S.H., Sabahi M., Niapour S.M. Modeling and Control of a New Three-Input DC-DC Boost Converter for Hybrid PV/FC/Battery Power System. IEEE Transactions on Power Electronics, 2012, no. 27/5, pp. 2309-2324.

11. SHIM-kontrollery. Texas Instruments [PWM controllers of Texas Instruments]. Available at: https://www.compel.ru/lib/ne/ 2007/3/5-shim-kontrolleryi-texas-instruments (accessed 11 February 2018).

\section{Information about the authors}

Yury V. Krasnobaev, Dr. Sc., professor, Siberian Federal University.

Oleg V. Nepomnyashchiy, Cand. Sc., professor, Siberian Federal University.

Vladimir I. Ivanchura, Dr. Sc., professor, Siberian Federal University.

Irina N. Pozharkova, Cand. Sc., associate professor, FSBEE HE Siberian Fire and Rescue Academy EMERCOM of Russia; associate professor, Siberian Federal University.

Aleksey P. Yablonskiy, undergraduate, Siberian Federal University.
12. Shtrapenin G. New generation of integrated circuits. National Semiconductor for switching power supplies. Components and technologies, 2007, no. 8, pp. 114-118. In Rus.

13. Analogovy mir Maxim. Novye mikroskhemy pitaniya [Analog world of Maxim. New power microcircuits]. Moscow, Maxim Integrated Products Publ., 2014. No. 3, 60 p.

14. Wen Y., Trescasesr 0. DC-DC Converter With Digital Adaptive Slope Control in Auxiliary Phase for Optimal Transient Response and Improved Efficiency. IEEE Transactions on Power Electronics, 2012, no. 27/7, pp. 3396-3409.

15. Zhou G., Xu J., Jin Y. Improved digital peak current predictive control for switching DC-DC converters. IET Power Electronics, 2011, no. 4/2, pp. 227-234.

16. Vintonyak N.P., Fedotov V.A., Semenov V.D. Technique of designing microcontroller software for high-speed control systems. Reports of TUSUR, 2015, no. 2, pp. 175-179. In Rus.

17. Mahdavikhah B., Jain P., Prodic A. Digitally controlled multiphase buck-converter with merged capacitive attenuator. TwentySeventh Annual IEEE Applied Power Electronics Conference and Exposition (APEC). Orlando, 2012. pp. 1083-1087.

18. Soustin B.P., Ivanchura V.I., Chernyshev A.I., Islyaev Sh.N. Sistemy elektropitaniya kosmicheskikh apparatov [Power supply systems for space vehicles]. Novosibirsk, Nauka Publ., 1994. 318 p.

19. Tsypkin Ya.Z. Teoriya lineynykh impulsnykh system [Theory of linear impulse systems]. Moscow, Fizmatlit Publ., 1963. 968 p.

20. Manakov A.V., Ivanchura V.I., Soustin B.P. Synthesis and investigation of a high-speed switching voltage regulators with PWM. Technical electrodynamics, 1987, no. 1, pp. 43-51.

21. Krasnobaev Yu.V. Methodology of synthesis of laws and structures of converter control devices. University proceedings. Ser. Instrument engineering, 2004, vol. 47, no. 4, pp. 39-48. In Rus.

Received: 24 April 2018. 